Research Paper

\title{
Thrombopoietin could protect cerebral tissue against ischemia-reperfusion injury by suppressing NF-KB and MMP-9 expression in rats
}

\author{
Wenjuan $\mathrm{Wu}^{1,2}$,Wei Zhong1 ${ }^{1}$, Bing Lang ${ }^{3}$, Zhiping $\mathrm{Hu}^{1}$, Jialin $\mathrm{He}^{1}$, Xiangqi Tang ${ }^{\circledR}$ \\ 1. Department of Neurology, The Second Xiangya Hospital, Central South University, Changsha, Hunan 410011, China \\ 2. Department of Neurology, The First Affiliated Hospital of Henan University of Science and Technology \\ 3. National Clinical Research Center for Mental Disorders, the Second Xiangya Hospital, Central South University, Changsha, Hunan 410011, China \\ Wenjuan Wu and Wei Zhong contributed equally to this work
}

$\triangle$ Corresponding author: Xiangqi Tang, Department of Neurology, The Second Xiangya Hospital, Central South University, Renmin Road 139\#, Changsha, Hunan 410011, China. Tel: +86 13875807186; Fax: 0731-84896038; Email: txq6633@csu.edu.cn

(c) Ivyspring International Publisher. This is an open access article distributed under the terms of the Creative Commons Attribution (CC BY-NC) license (https://creativecommons.org/licenses/by-nc/4.0/). See http://ivyspring.com/terms for full terms and conditions.

Received: 2018.05.29; Accepted: 2018.07.26; Published: 2018.08.10

\begin{abstract}
Objective: To determine the neuroprotective effects and underpinning mechanisms of thrombopoietin (TPO), Matrix Metalloproteinase-9(MMP-9) and Nuclear Factor-KB (NF-KB) after focal cerebral ischemia-reperfusion in rats.

Methods: Male rats underwent 2 hours of right middle cerebral artery occlusion (MCAO) followed by 22 hours of reperfusion. PBS or TPO $(0.1 \mathrm{ug} / \mathrm{kg})$ was administered from caudal vein before reperfusion. Neurologic deficits, brain edema, Evans blue (EB) extravasation, NF-KB and MMP-9 expression were subsequently examined.

Results: Ischemia-reperfusion injury produced a large area of edema. TPO significantly reduced edema and alleviated neurologic deficits after ischemia-reperfusion. Ischemia-induced increases of NF- $k B$, MMP-9 and Evans blue extravasation were reduced by TPO intervention.

Conclusion: TPO improved neurological function and ameliorated brain edema after stroke, partly by reducing the ischemia-induced increase of NF-KB and MMP-9.
\end{abstract}

Key words: Thrombopoietin (TPO); Nuclear factor-kB (NF-kB); Matrix Metalloproteinase-9 (MMP-9); Ischemia-Reperfusion (IR)

\section{Introduction}

The most effective treatment for ischemic stroke is thrombolytic intervention. However, the process of thrombolytic intervention is accompanied with cerebral ischemia-reperfusion injury, leading to increased permeability of blood-brain barrier (BBB) and brain edema [1]. Reperfusion injury is attributed to various factors, including inflammation, oxidative stress and proteolytic enzyme, which result in damage of BBB integrity and hemorrhagic transformation [2]. It has to be noted that recombinant tissue plasminogen activator(r-tPA) can only be administered within 3 hours after stroke [3]. Therefore, it is important to look for alternative therapy for ischemic stroke, especially for the cases with brain ischemia longer than 3 hours.

Increasing evidence has suggested that hematopoietic growth factors are a new treatment strategy for stroke. Hematopoietic growth factors are proteins that regulate the production of blood cells. However, these factors can manifest additional functions beyond their hematopoietic action. Thrombopoietin (TPO) is a primary hematopoietic growth factor for platelet production, participating in the process of hematopoietic cell's proliferation, differentiation and maturation [4]. TPO is successfully used for thrombocytopenia. In addition to the hematopoietic system, TPO and its receptors (i.e. c-MPL) are expressed in various organs including 
heart and nervous system which indicates that TPO may have hematopoiesis-independent effects [5]. TPO can augment angiogenic response [6], improve ventricular function and present protection against myocardial ischemia [7]. Besides its expression in hematopoietic system, TPO receptor (c-MPL) also located in the central nervous system, and can inhibit the apoptosis of nerve cells, through the activation of the PI-3K/AKT signal pathway [8]. In contrast, Balcik showed that increased TPO level may multiply both platelet count and size, contributing to the progress of ischemic stroke [9]. Research has also shown that during moderate hypoxia ischemia, TPO promotes the apoptosis of nerve cell, whereas under severe hypoxia ischemia condition, TPO inhibits the apoptosis of nerve cell [10]. Therefore, how ischemia alters the expression of TPO still remain debatable and its roles during ischemia-reperfusion are far from clear.

Inflammation plays an important role in the damage of blood brain barrier after ischemicreperfusion injury $[11,12]$. NF-kB is a vital regulator of inflammation response and nerve cell apoptosis. Interestingly, TPO has been demonstrated to regulate PI-3K signal pathway and phosphorylation of PI-3K signal pathway could activate NF-KB and MMP-9 [13-15]. Inhibition of stroke-induced increase of NF- $\mathrm{kB}$ could protect brain from cerebral ischemiareperfusion injury [16]. MMP-9 is an independent factor of BBB damage. Several studies showed that up-regulated expression of MMP-9 could degrade extracellular matrix and intercellular tight junction, leading to increased permeability of BBB and subsequent brain edema and hemorrhagic transformation after ischemia-reperfusion [17].

It is still unknown whether TPO could protect against cerebral ischemia reperfusion injury or not, therefore, the purpose of our study is to investigate the action and mechanism of TPO in model of stroke. We propose that TPO regulates the expression of NF-KB and MMP-9 and thus modulates the permeability of BBB after ischemia-reperfusion injury.

\section{Materials and methods}

\subsection{Animal model and groups}

The research was conducted in accordance with the Guide for Care and Use of Laboratory Animals by the United National Institutes of Health. All experimental protocols were approved by the Second Xiangya Hospital Animal Care Committee of Central South University. Male rats weighing from $250 \mathrm{~g}$ to $300 \mathrm{~g}$ were divided randomly into three groups: the sham operation group, ischemia-reperfusion model (IR) group and TPO intervention group.
The model of focal cerebral ischemia-reperfusion was established in Sprague-Dawley rats as per the Longa's suture method [18]. A silicone-coated nylon monofilament was passed through the bifurcation of the common carotid artery to the internal carotid artery, advancing along the internal carotid artery to approximately $18-22 \mathrm{~mm}$ from the bifurcation until a proximal occlusion of the right middle cerebral artery was established. After 2 hours of occlusion, the filament was withdrawn slowly to allow blood supply in the middle cerebral artery for 22 hours. Room temperature was maintained at $25^{\circ} \mathrm{C}$ during the operation. The body temperature was maintained until recovery.

Sham operation group only received the internal carotid artery separation, whilst IR group and TPO intervention group received 2 hours of $\mathrm{MCAO}$ followed by 22 hours of reperfusion, PBS $(0.1 \mathrm{ug} / \mathrm{kg})$ or TPO $(0.1 \mathrm{ug} / \mathrm{kg})$ was injected via tail vein respectively prior to reperfusion. Each group was randomly divided into subgroups, for TTC staining, brain water content measuring, Evans blue dyeing, $\mathrm{HE}$ and immunohistochemistry staining, Western blot and RT-PCR.

\subsection{Neurological deficits score}

The neurological deficits were evaluated by an examiner blind to the experimental groups, after 2 hours of MCAO and 22 hours of reperfusion [18]. Details were as follows: 0 , no symptoms of neural damage; 1 , failure to extend left forepaws; 2 , circling to the left; 3 , falling down to the left; 4 , no spontaneous walking with a loss of consciousness. Rats received 1 to 3 score were selected as observational objectives.

\subsection{Triphenyltetrazolium chloride staining}

Triphenyltetrazolium chloride (TTC; Sigma) staining was used for confirming the success of the MCAO model. After 22 hours of reperfusion, the rats were deeply re-anesthetized by $10 \%$ chloral hydrate. The brain tissue were quickly removed on ice and placed in an environment of $-20^{\circ} \mathrm{C}$ for half an hour. Then the brain tissue were sliced into 6 coronal sections of $2 \mathrm{~mm}$ thickness and immersed in 2\% TTC solution in the dark at $37^{\circ} \mathrm{C}$ for $30 \mathrm{~min}$ and kept in $4 \%$ paraformaldehyde at $4^{\circ} \mathrm{C}$ overnight.

\subsection{Brain water content}

Rats were killed after 22 hours of reperfusion under deeply anesthetized. Brain tissues were split into right and left hemispheres without olfactory bulb, cerebellum and brain stem, the right hemispheres were evaluated wet weights $(\mathrm{ww})$ on a balance immediately, and then dried in the oven at $110^{\circ} \mathrm{C}$ for 24 hours to get the dry weights $(\mathrm{dw})$. The 
brain water content was calculated with the following formula: brain water content $=(w w-d w) / w w \times 100 \%$.

\subsection{Evans Blue extravasation}

Rats were injected with Evans blue (2\% in saline, $4 \mathrm{~mL} / \mathrm{kg}$; Sigma) via the tail vein 1 hour prior to sacrifice. Before decollation, rats were perfused with saline to remove the intravascular dye. Then the brain tissue were homogenized in $2 \mathrm{~mL} 50 \%$ trichloroacetic acid and centrifuged at $10,000 \mathrm{rpm}$ for 30 minutes. The supernatant liquid $(50 \mathrm{uL})$ were mixed with ethanol (150ul) and measured absorbance (at 632nm) by spectrophotometry. The content of Evans blue was quantified with a standard curve and expressed as ug Evans blue / $g$ brain tissue.

\subsection{HE and Immunohistochemistry staining}

After 22 hours of reperfusion, the brains were removed after cardiac perfusion with saline and paraformaldehyde $(4 \%)$. Then the brains were post-fixed in $4 \%$ paraformaldehyde for 24 hours, embedded in paraffin and cut into $4 \mu \mathrm{m}$ thick serial co ronal sections after the optic chiasm. Sections were baked in the oven at $60^{\circ} \mathrm{C}$ for 90 minutes, dewaxed in xylene and hydrated in graded ethanol. HE staining was used for observing the profile of cerebral cortex tissue and immunohistochemical staining was used to examine the expression of MMP-9 (1:100) and NF- $\mathrm{kB}$ $(1: 50)$ in the ischemic hemisphere. Five different visual fields were chosen randomly from each slice and three slices were used from each sample. Images were taken under an objective lens of $40 \mathrm{x}$ and were collected by microscope-digital photographic system (DP12 SZX7, Olympus Inc., Japan).

\subsection{Western Blot}

Ischemic and control brain tissues were homogenized with RIPA buffer (Applygen, China) which contained a mixture of protease inhibitors, and the protein concentration of extracts were determined by BCA protein analysis kit (Pierce, Rockford, USA). Protein extracts (50 $\mathrm{\mu g}$ of total protein) were seperated in $10 \%$ sodium dodecyl sulfate-polyacrylamide gels, then transferred to nitrocellulose membranes. Membranes were incubated with TBS-T (tris-buffered saline and $0.1 \%$ Tween-20, Sigma, USA) containing $5 \%$ non-fat milk at room temperature for 1 hour, then with primary antibodies against NF-kB (1:200 dilution, Santa Cruz) and MMP-9(1:1000 dilution, Abcam) at $4^{\circ} \mathrm{C}$ for a whole night. Membranes loaded with primary antibodies were washed with TBS-T for 3 times, and then incubated for 1 hour at room temperature with horseradish peroxidase conjugate secondary antibodies (1:3000 dilution, Santa Cruz). The membranes were then developed with the
SuperEnhanced chemiluminescence detection kit (Thermo pierce, USA). The membranes were incubated with $\beta$-actin primary antibodies (1:4000 dilution, Sigma) as control. Protein expression was standardized with an equivalent $\beta$-actin protein. The bands were detected using X-ray film and quantified using Quantity-one Analysis software.

\subsection{Real-Time PCR}

Frozen brain tissues were homogenized with Trizol reagent at the ratio of $100 \mathrm{mg} / 5 \mathrm{ml}$. Total RNA was extracted following technical manual of Trizol RNA kit (Invitrogen, USA). The content and purity of extracted RNA were determined by nucleic acid protein spectrophotometer, the ratio of $260 / 280 \mathrm{~nm}$ absorbance was 1.8-2.0. Extracted RNA was electrophoresed with $1 \%$ of agarose gel to display clear rRNA bands for the template quality and purity control, and then reversely transcribed in accordance with instructions of the SuperRT First-strand cDNA synthesis kit (ComWin, China). Reverse transcription products were amplified by SYBER ${ }^{\circ}$ Green PCR Master Mix system (Invitrogen, USA) in 10ul final reaction volume. Relative abundance of mRNA was calculated after normalization with $\beta$-actin. RT-PCR was used for analyzing the levels of NF-kB and MMP-9 mRNA after 22 hours of reperfusion. The mean $\mathrm{Ct}$ values were normalized by the internal control $\beta$-actin. The difference of $\Delta \mathrm{Ct}$ values of the control sample was calculated and defined as $\Delta \Delta \mathrm{Ct}$. The relative value of mRNA expression level was expressed as $2-\Delta \Delta \mathrm{Ct}$.

The primers were as follows: NF-kB: F5'-GGTGGAGTTTGGGAAGGATTTG-3', R5'-TTTT CTCCGAAGCTGAACAAACAC-3'; MMP-9:F5'-GGC ACCATCATAACATCACCTA-3', R5'-GACACCAAA CTGGATGACAATG-3'; $\beta$-actin: F5'-CATCCTGCGTC TGGACCTGG-3'， R5'-TAATGTCACGCACGATTTC C-3'.

\subsection{Statistical Analysis.}

Statistical analysis was performed with SPSS version 19.0. Data were expressed as mean $\pm S D$, and analyzed with ANOVA, followed by the StudentNewman-Keuls test, but neurological deficit assessment was tested by Mann-Whitney $U$ test between two groups. The significance level was set at $\mathrm{P}<0.05$.

\section{Results}

\subsection{TTC staining}

No infarction was observed in the sham operation group, while white infarct lesion appeared in the IR group and TPO intervention group, verifying successful establishment of MCAO model in 
rats (Figure 1A).

\subsection{Neurological deficit score}

Neurological deficits were assessed and scored within 24 hours after ischemia. There were no symptoms of neurological deficit in the sham operation group. Compared with IR group, the neurological deficit scores were significantly reduced in TPO intervention group (Table 1, Figure 1B).

\subsection{TPO reduced brain edema and BBB damage}

The brain water content of IR group was higher than that in sham operation group, TPO intervention could reduce the increase of brain water content caused by IR injury (Table 2, Figure 1C).

BBB disruption following 2 hours ischemia and 22 hours reperfusion was assessed by measuring the content of Evan's blue in brain tissue. The content of Evan's blue in ischemic hemisphere is significantly higher than that in the contralateral hemisphere in IR group. Compared with the IR group, TPO intervention group showed significantly less Evan's blue content (Table 3, Figure 1D).

\subsection{TPO attenuated ischemia-induced}

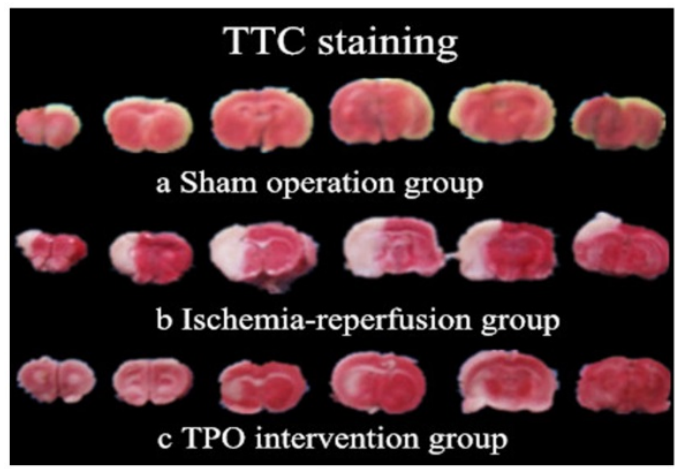

A

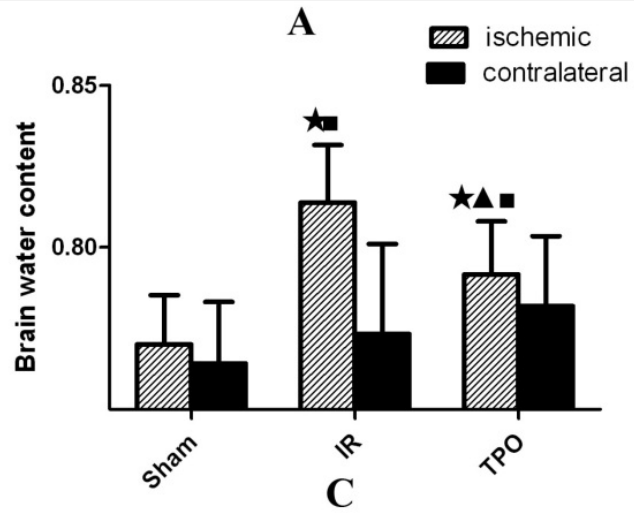

\section{neuronal cell damage}

The cell morphology and structure were normal in sham operation group, but presented obvious changes in IR and TPO intervention group. In the ischemia and infarction region of brain tissue, the gross brain structures disappeared with the neuronal numbers decreased. Some neurons presented karyopyknosis. TPO intervention could reduce neuronal damage comparing with IR group (Figure 2A).

\subsection{TPO suppressed the expression of MMP-9 and NF-KB}

We further performed immunohistochemistry in order to determine the expressing levels of MMP-9 and NF- $\mathbf{K B}$ in the cerebral cortex infarction region. MMP-9 and NF-KB positive staining were mainly distributed in neurons, endothelial cells and neutrophils. MMP-9 protein was located in cytoplasm, while NF-kB was located both at cytoplasm and nucleus. Five fields were chosen randomly from each slice, then the average positive cell number were calculated by the sum divided by five, then the average cell number was calculated from the three slices. Few positive cells were found in sham operation group, but there was a considerable
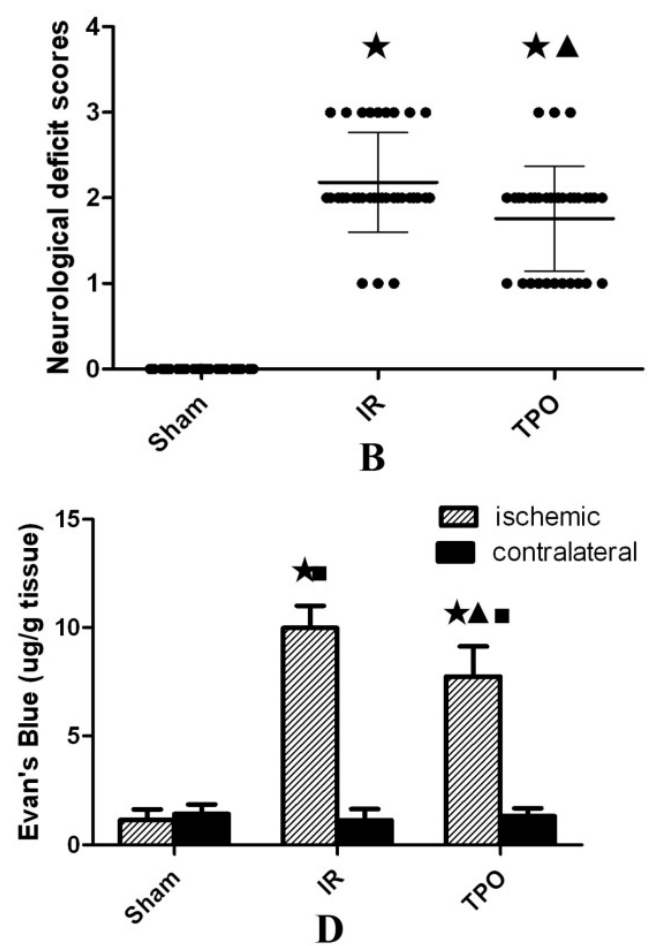

Figure 1. Effect of thrombopoietin in ischemia-reperfusion rats' brains. (A) TTC staining of brain slices after ischemia- reperfusion. Uniformly red region in Sham operation group (a) vs. white infarct lesion in IR group (b) and TPO intervention group(c). (B) Compared with sham operation group, the neurological deficit scores were significantly increased in IR and TPO intervention group, and TPO intervention decreased neurological deficit at 24h after MCAO. (C) The brain water content of ischemic hemispheres was significantly increased after MCAO, and TPO intervention reduced ischemia-induced increase of brain water content, whereas no difference was found in contralateral hemispheres. (D) The Evan's blue extravasation of ischemic hemispheres is significantly higher than that of sham group and contralateral hemispheres in IR and TPO intervention group, and TPO intervention reduced Evan's blue content significantly compared with the IR group. ( ${ }^{\star}$ Compared with the sham operation group, P $<0.05 ;{ }^{\wedge}$ Compared with the IR group, $\mathrm{P}<0.05$; "Compared with the contralateral brain, $\mathrm{P}<0.05$ ) 
increase in the number of positive cells in IR group, TPO intervention could reduce the increased expression of MMP-9 and NF-kB caused by ischemia (Figure 2B, 2C, 3C and Table 4).

Table 1. Neurological deficit score of each group $(n=33, \bar{X} \pm S)$

\begin{tabular}{ll}
\hline Group & Grade \\
\hline Sham operation group & 0 \\
IR group & $2.18 \pm 0.58^{\star}$ \\
TPO intervention group & $1.76 \pm 0.61^{\star} \mathbf{\Delta}$ \\
\hline
\end{tabular}

${ }^{\star}$ Compared with the sham operation group, $\mathrm{P}<0.05 ; \mathbf{\Delta}$ Compared with the IR group, $\mathrm{P}<0.05$

Table 2. The brain water content of each group $(n=6, \bar{X} \pm S)$

\begin{tabular}{lll}
\hline Group & Ischemic hemisphere & Contralateral hemisphere \\
\hline Sham operation group & $0.77 \pm 0.015$ & $0.76 \pm 0.019$ \\
IR group & $0.81 \pm 0.018^{\star \star}$ & $0.77 \pm 0.028$ \\
TPO intervention group & $0.79 \pm 0.016^{\star \Delta}$ & $0.78 \pm 0.022$
\end{tabular}

${ }^{\star}$ Compared with the sham operation group, $\mathrm{P}<0.05 ; \mathbf{\Delta}$ Compared with the IR group, $\mathrm{P}<0.05$.

-Compared with the contralateral brain, $\mathrm{P}<0.05$

Table 3. The Evan's blue content of each group $(n=6, \bar{X} \pm S$, ug/g)

\begin{tabular}{lll}
\hline Group & Ischemic hemisphere & Contralateral hemisphere \\
\hline Sham operation group & $1.15 \pm 0.49$ & $1.42 \pm 0.44$ \\
IR group & $9.98 \pm 1.02^{\star} \boldsymbol{\star}_{\boldsymbol{*}}$ & $1.13 \pm 0.51$ \\
TPO intervention group & $7.74 \pm 1.39^{\star} \mathbf{\Delta .}$ & $1.32 \pm 0.36$
\end{tabular}

${ }^{\star}$ Compared with the sham operation group, $\mathrm{P}<0.05$; $\wedge$ Compared with the IR group, $\mathrm{P}<0.05$

-Compared with the contralateral brain, $\mathrm{P}<0.05$
Table 4. Number of MMP-9 and NF-KB positive cells in each group $(n=6, \bar{X} \pm S)$

\begin{tabular}{lll}
\hline Group & MMP-9 & NF-kB \\
\hline Sham operation group & $9.31 \pm 2.96$ & $7.17 \pm 1.98$ \\
IR group & $30.78 \pm 5.79 \mathbf{\Delta}$ & $40.82 \pm 8.73 \mathbf{\Delta}$ \\
TPO intervention group & $22.41 \pm 4.58 \mathbf{\Delta .}$ & $24.67 \pm 6.33 \mathbf{\Delta .}$ \\
\hline $\mathbf{A}$ Compared with the sham operation group, $\mathrm{P}<0.05 ;$ - Compared with the IR group, \\
$\mathrm{P}<0.05$
\end{tabular}

Table 5. Relative protein level of MMP-9 and NF-KB in each group $(n=6, \quad \bar{X} \pm S)$

\begin{tabular}{|c|c|c|}
\hline Group & MMP-9 & NF-kB \\
\hline Sham operation group & $0.17 \pm 0.059$ & $0.23 \pm 0.033$ \\
\hline IR group & $0.37 \pm 0.021 \triangleleft$ & $0.49 \pm 0.099 \Delta$ \\
\hline TPO intervention group & $0.25 \pm 0.058 \wedge$ • & $0.32 \pm 0.068 \wedge$ \\
\hline
\end{tabular}
$\mathrm{P}<0.05$

We further quantified the protein level of MMP-9 and NF-KB with western blot. The expression of MMP-9 and NF-kB proteins were up-regulated after ischemia-reperfusion. TPO intervention significantly decreased the MMP-9 and NF-KB protein levels (Figure 3A, 3B and Table 5). In line with the results of immunohistochemistry and Western blot, the mRNA expression of MMP-9 and NF- $\mathrm{kB}$ was up-regulated after ischemia-reperfusion. The augmented expression of MMP-9 and NF-kB was remarkably attenuated in TPO intervention group (Figure 3D and Table 6).

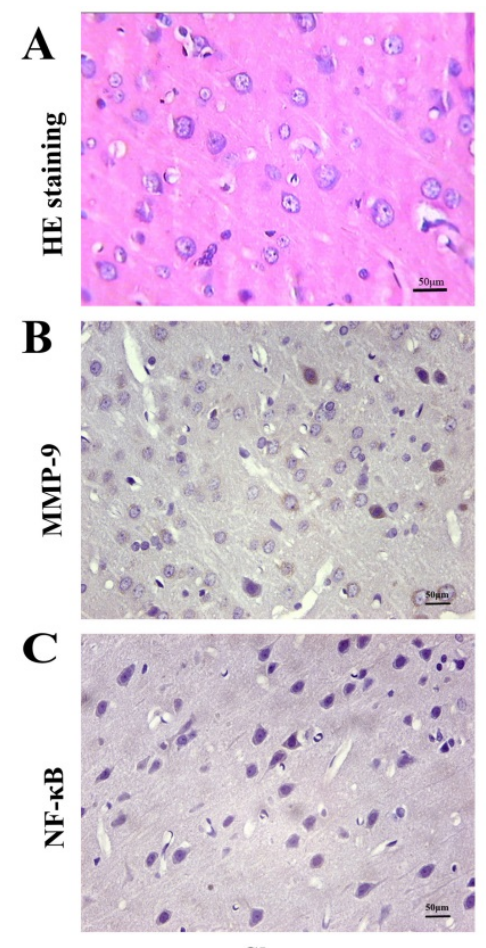

Sham

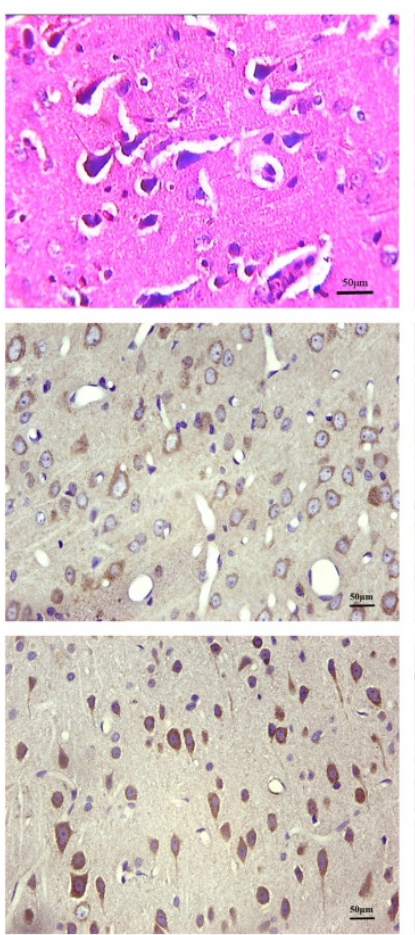

IR

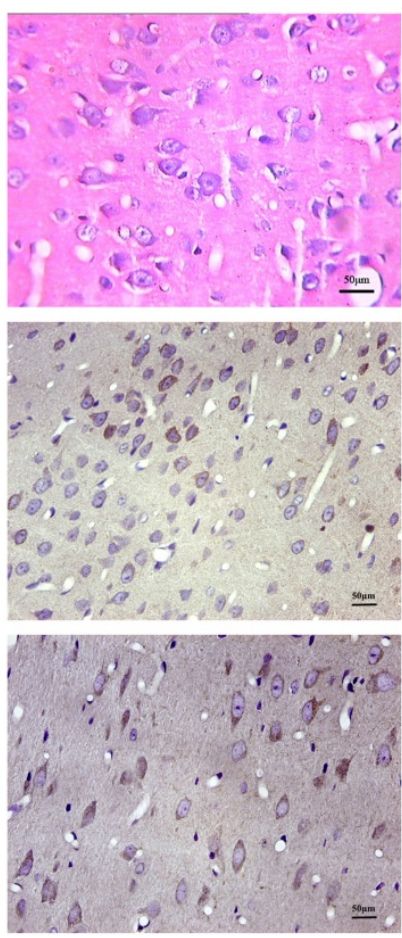

TPO

Figure 2. Hematoxylin-Eosin staining (A) and Immunohistochemical staining of MMP-9 (B) and NF-KB (C) in the cerebral cortex. Scale bar, 50 $\mu m$. (400 $\times$ magnification) There was increased MMP-9, NF-KB immunoreactivity after Ischemia-reperfusion. TPO intervention reduced MMP-9, NF-KB immunoreactivity significantly. 

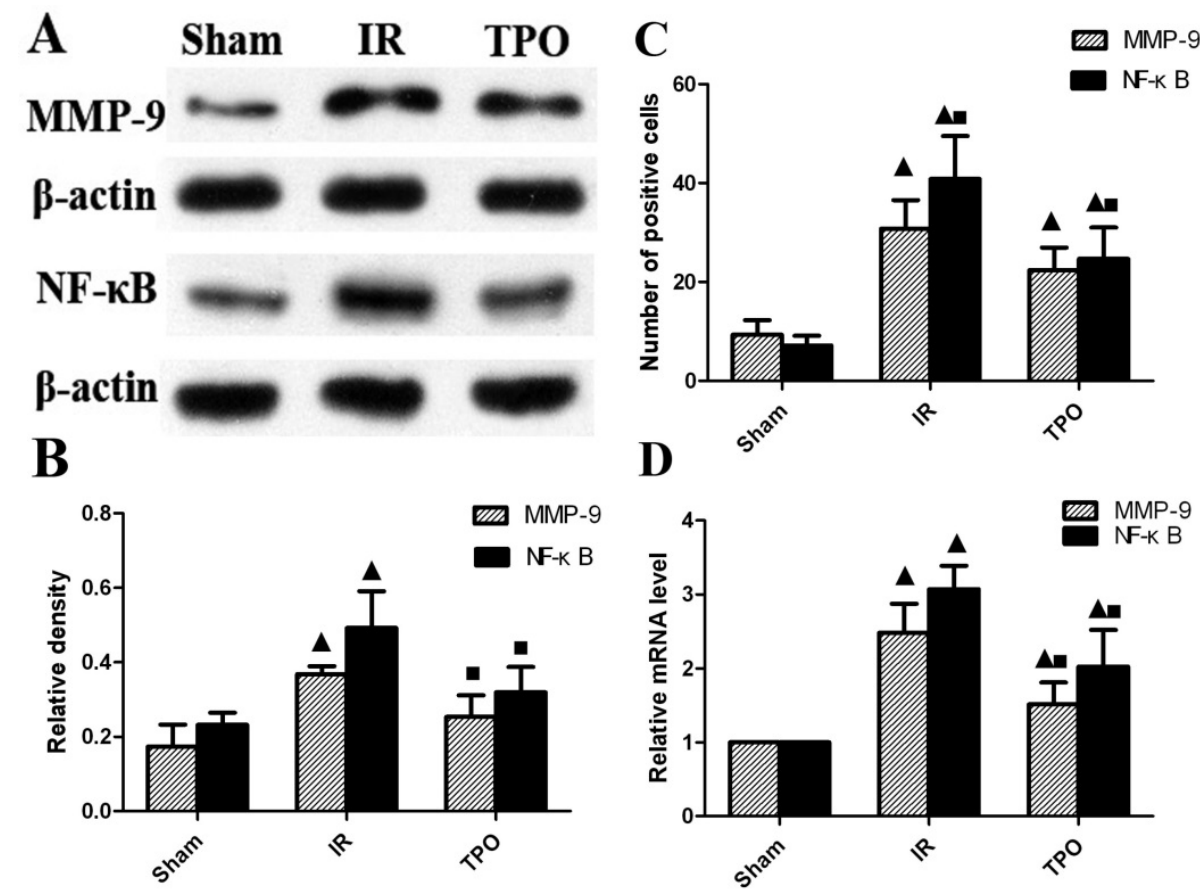

Figure 3. TPO suppressed the expression of MMP- 9 and NF-KB. (A, B) Western blot results showing the expressing levels of MMP-9 and NF-KB protein; (C) Number of MMP-9 and NF-KB positive cells assessed by immunohistochemistry; (D) RT-PCR results displaying the mRNA expression of MMP-9 and NF-KB. The expression of MMP-9 and NF-KB was massively increased in IR group, and TPO intervention effectively blocked the increase caused by ischemia-reperfusion. $\left({ }^{\boldsymbol{\Lambda}}\right.$ Compared with the sham operation group, $\mathrm{P}<0.05$; "Compared with the IR group, $\mathrm{P}<0.05$ )

Table 6. Relative mRNA expression of MMP-9 and NF-KB in each $\operatorname{group}(n=6, \bar{X} \pm S)$

\begin{tabular}{lll}
\hline Group & MMP-9 & NF-KB \\
\hline Sham operation group & 1 & 1 \\
IR group & $2.49 \pm 0.39 \Delta$ & $3.07 \pm 0.32 \Delta$ \\
TPO intervention group & $1.52 \pm 0.30 \Delta$ & $2.03 \pm 0.49 \mathbf{~ - ~}$ \\
\hline
\end{tabular}

$\triangle$ Compared with the sham operation group, $\mathrm{P}<0.05$; -Compared with the IR group, $\mathrm{P}<0.05$

\section{Discussion}

Thrombopoietin, the main regulator in the generation of megakaryocyte and platelet production, used to be a common treatment for thrombocytopenia [19]. In recent years, a large number of studies have also found that TPO participates in some important physiological processes beyond the hematopoietic system, especially in nervous system. Whether it is beneficial or harmful after ischemia is unknown. Previous study has shown that TPO and mean platelet volume were increased significantly in stroke patients, leading to better hemostatic tendency, which might contribute to the progress of ischemia stroke [9]. Decreased platelet count caused by TPO inhibitor could reduce experimental occlusive thrombogenesis, suggesting that suppressing platelet production with TPO inhibitor may prevent stroke and heart attack [20]. However, other studies had opposite outcomes. Baker et al. showed that TPO treatment could reduce myocardial infarct size and apoptosis following ischemia-reperfusion through multiple signaling pathways including JAK-2 and p42/44 MAPK as well as K (ATP) channels [21]. TPO also enhanced the correction of ischemia via promoting angiogenic response mediated by the increased platelet level [6]. The present study shows that after 2 hours ischemia followed by 22 hours reperfusion, the BBB permeability was massively increased, leading to severe brain edema and neurologic deficit, TPO intervention significantly reduced brain edema and neurologic deficit caused by ischemia-reperfusion injury. These results suggest that TPO could protect brain from ischemia-reperfusion injury, and hopefully be a new therapeutic method in stroke.

The MMP family members, especially MMP-9, have been shown significantly up-regulated after stroke, degrading tight-junction proteins and leading to damage of BBB [22]. Higher activity and expression of MMP-9 were closely related to brain edema [23]. Inhibition of MMP-9 expression can relieve cerebral edema after stroke [24]. Zhou et al. showed that TPO could reduce ischemic brain injury by inhibiting the stroke-induced increase in MMP-9[25]. They found that TPO significantly inhibited the stroke-induced increase in MMP-9 mRNA expression, active-MMP-9 protein expression, and MMP-9 enzymatic activity. In stroke, the MMP-9 activation was of multi-factorial origins including inflammation [26]. NF-kB promoted inflammation process and activated inflammatory cytokines, resulting in inflammation cascade amplification [14, 27]. NF-kB phosphorylation 
induced by TNF-a or PI3K- $\gamma$ could damage endothelial cell, leading to BBB damage and brain edema [27, 28]. Guan et al. found that Ruscogenin protected against brain ischemia by inhibiting NF-KB p65 expression [16], which also meant inhibition of NF-kB expression could protect brain tissue from IR injury. There was NF-kB transcriptional regulation binding site before TATA box of the gene regulation sequence of MMP-9. Activated NF-kB could increase the transcription of MMP-9[29]. Annabi found that inhibiting the NF-kB phosphorylation by resveratrol could decrease MMP-9 expression and BBB damage [30]. Therefore, we hypothesize that TPO could regulate NF-kB/MMP-9 to protect brain from ischemia-reperfusion injury. The present study shows that the expression of NF-KB and MMP-9 was significantly increased in brain tissue after ischemia reperfusion, which is in line with their mRNA expression, TPO treatment obviously reduced the stroke-induced increase of NF-kB and MMP-9.

Ehrenreich et al. showed that TPO and its receptor were down-regulated upon hypoxia, could cause death of cultured hippocampal neurons at low concentration, and lost its death-promoting function at high concentration [31]. In this study, the TPO intervention was at the dose of $0.1 \mathrm{ug} / \mathrm{kg}$, in accordance with Zhou's results, which demonstrated that $0.1 \mathrm{ug} / \mathrm{kg}$ is most effective to reverse ischemia-reperfusion injury [25]. Additionally, decreasing the platelet production, but remaining at a physiological range and without interfering by the hemostatic function of platelets, has been suggested as a safe alternative to platelet inhibitors for thromboprophylaxis [20].

Besides, our research had some limitations. Firstly, we didn't conduct detection of active form of NF-kB. Secondly, we didn't perform matrix metalloproteinase zymography to determine whether the activity of MMP-9 has altered by TPO intervention. We will conduct relevant activity testing of NF-kB and MMP-9 in the further study. Thirdly, As the present study didn't measure the count and the function of platelet in blood, future studies shall focus on the balance between brain protective and platelet promoting function.

\section{Conclusion}

In summary, this study shows that the intervention of TPO can significantly reduce neurologic deficit, improve the BBB permeability and relieve cerebral edema, suggesting that the TPO might have the neuro-protective effects in ischemia reperfusion injury via decreasing the expression of MMP-9 and NF-kB. This experiment reveals that the NF-kB / MMP-9 signaling pathway may be involved in the BBB damage after cerebral ischemia reperfusion injury and thus provides a new treatment strategy for stroke.

\section{Acknowledgements}

This work was supported by the National Natural Science Foundation of China (Grant no.81271298), the Hunan Provincial Science and Technology Department in China (Grant no.2011SK3236).

\section{Competing Interests}

The authors have declared that no competing interest exists.

\section{References}

1. Zhang Q, Gao T, Luo Y, et al. Transient focal cerebral ischemia/reperfusion induces early and chronic axonal changes in rats: its importance for the risk of Alzheimer's disease. PLoS One 2012;7:e33722

2. Tu Q, Cao H, Zhong W, et al. Atorvastatin protects against cerebral ischemia/reperfusion injury through anti-inflammatory and antioxidant effects. Neural Regen Res 2014;9:268-275

3. Boudreau DM, Guzauskas GF, Chen E, et al. Cost-effectiveness of recombinant tissue-type plasminogen activator within 3 hours of acute ischemic stroke: current evidence. Stroke 2014;45:3032-3039

4. Vainchenker W, Besancenot R, Favale F. Megakaryopoiesis: regulation of platelet production by thrombopoietin. Bull Acad Natl Med 2013;197:395-406

5. Alvarez RM, Fernandez BI, Arias-Salgado EG, et al. Effects of thrombopoietin receptor agonists on procoagulant state in patients with immune thrombocytopenia. Thromb Haemost 2014;112:65-72

6. Kawamoto T, Sasajima J, Sugiyama Y, et al. Ex vivo activation of angiogenic property in human peripheral blood-derived monocytes by thrombopoietin. Int J Hematol 2013;98:417-429

7. Chan KY, Zhou L, Xiang P, et al. Thrombopoietin improved ventricular function and regulated remodeling genes in a rat model of myocardial infarction. Int J Cardiol 2013;167-2546-2554

8. Yang M, Shu LL, Cui Y. The role of PDGF/PDGFR in the regulation of platelet formation. Zhongguo Shi Yan Xue Ye Xue Za Zhi 2011;19:1097-1101

9. Balcik OS, Bilen S, Ulusoy EK, et al. Thrombopoietin and mean platelet volume in patients with ischemic stroke. Clin Appl Thromb Hemost 2013;19:92-95

10. Liu XM, Feng Y, Li AM. Effect of G-CSF and TPO on HIBD in neonatal rats. Asian Pac J Trop Med 2015;8:132-136

11. Yadav VR, Prasad S, Gupta SC, et al. 3-Formylchromone interacts with cysteine 38 in p65 protein and with cysteine 179 in IkappaBalpha kinase, leading to down-regulation of nuclear factor-kappaB (NF-kappaB)-regulated gene products and sensitization of tumor cells. J Biol Chem 2012;287:245-256

12. Huang L, Wong S, Snyder EY, et al. Human neural stem cells rapidly ameliorate symptomatic inflammation in early-stage ischemic-reperfusion cerebral injury. Stem Cell Res Ther 2014;5:129

13. Monzen S, Tashiro E, Kashiwakura I. Megakaryocytopoiesis and thrombopoiesis in hematopoietic stem cells exposed to ionizing radiation. Radiat Res 2011:176:716-724

14. Zhuang R, Lin MX, Song QY, et al. Effects of curcumin on the expression of nuclear factor-kappaB and intercellular adhesion molecular 1 in rats with cerebral ischemia-reperfusion injury. Nan Fang Yi Ke Da Xue Xue Bao 2009;29:1153-1155

15. Shih RH, Wang CY, Yang CM. NF-kappaB Signaling Pathways in Neurological Inflammation: A Mini Review. Front Mol Neurosci 2015;8:77

16. Guan T, Liu Q, Qian Y, et al. Ruscogenin reduces cerebral ischemic injury via NF-kappaB-mediated inflammatory pathway in the mouse model of experimental stroke. Eur J Pharmacol 2013;714:303-311

17. Takata F, Dohgu S, Matsumoto J, et al. Brain pericytes among cells constituting the blood-brain barrier are highly sensitive to tumor necrosis factor-alpha, releasing matrix metalloproteinase- 9 and migrating in vitro. J Neuroinflammation 2011;8:106

18. Longa EZ, Weinstein PR, Carlson S, et al. Reversible middle cerebral artery occlusion without craniectomy in rats. Stroke 1989;20:84-91

19. Arai $\mathrm{Y}$, Jo T, Matsui $\mathrm{H}$, et al. Comparison of up-front treatments for newly diagnosed immune thrombocytopenia -a systematic review and network meta-analysis. Haematologica 2018;103:163-171

20. Tucker EI, Marzec UM, Berny MA, et al. Safety and antithrombotic efficacy of moderate platelet count reduction by thrombopoietin inhibition in primates. Sci Transl Med 2010;2:37r-45r 
21. Baker JE, Su J, Hsu A, et al. Human thrombopoietin reduces myocardial infarct size, apoptosis, and stunning following ischaemia/reperfusion in rats. Cardiovasc Res 2008;77:44-53

22. Tang $\mathrm{X}$, Zhong $\mathrm{W}, \mathrm{Tu} \mathrm{Q}$, et al. NADPH oxidase mediates the expression of MMP-9 in cerebral tissue after ischemia-reperfusion damage. Neurol Res 2014;36:118-125

23. Bauer AT, Burgers HF, Rabie T, et al. Matrix metalloproteinase- 9 mediates hypoxia-induced vascular leakage in the brain via tight junction rearrangement. J Cereb Blood Flow Metab 2010;30:837-848

24. Lee K, Lee JS, Jang HJ, et al. Chlorogenic acid ameliorates brain damage and edema by inhibiting matrix metalloproteinase- 2 and 9 in a rat model of focal cerebral ischemia. Eur J Pharmacol 2012;689:89-95

25. Zhou J, Li J, Rosenbaum DM, et al. Thrombopoietin protects the brain and improves sensorimotor functions: reduction of stroke-induced MMP-9 upregulation and blood-brain barrier injury. J Cereb Blood Flow Metab 2011;31:924-933

26. Ludewig P, Sedlacik J, Gelderblom M, et al. Carcinoembryonic antigen-related cell adhesion molecule 1 inhibits MMP-9-mediated blood-brain-barrier breakdown in a mouse model for ischemic stroke. Circ Res 2013;113:1013-1022

27. He W, Chen $\mathrm{W}$, Zhou $\mathrm{Y}$, et al. Xanthotoxol exerts neuroprotective effects via suppression of the inflammatory response in a rat model of focal cerebral ischemia. Cell Mol Neurobiol 2013;33:715-722

28. Jin R, Song Z, Yu S, et al. Phosphatidylinositol-3-kinase gamma plays a central role in blood-brain barrier dysfunction in acute experimental stroke. Stroke 2011;42:2033-2044

29. Chiu PS, Lai SC. Matrix metalloproteinase-9 leads to claudin-5 degradation via the NF-kappaB pathway in BALB/c mice with eosinophilic meningoencephalitis caused by Angiostrongylus cantonensis. PLoS One 2013;8:e53370

30. Annabi B, Lord-Dufour S, Vezina A, et al. Resveratrol Targeting of Carcinogen-Induced Brain Endothelial Cell Inflammation Biomarkers MMP-9 and COX-2 is Sirt1-Independent. Drug Target Insights 2012;6:1-11

31. Ehrenreich H, Hasselblatt M, Knerlich F, et al. A hematopoietic growth factor, thrombopoietin, has a proapoptotic role in the brain. Proc Natl Acad Sci U S A 2005;102:862-867 